\title{
Problems of Designing LDMOS-Transistors Working at Increased Supply Voltage
}

\author{
A.A. Glushko ${ }^{1,2}$, S.I. Babkin ${ }^{2}$, A.V. Amirkhanov ${ }^{2}$, L.A Zinchenko ${ }^{1}$, V.V. Makarchuk ${ }^{1,2}$ \\ ${ }^{1}$ Bauman Moscow State Technical University \\ ${ }^{2}$ Scientific Research Institute of System Analysis
}

\begin{abstract}
The design issues of LDMOS transistors, oriented to work at $+12 \mathrm{~V}$ supply voltage, are considered. Particular attention is paid to the determination of the impurity concentration in the DRIFT-region of doping to achieve the maximum breakdown voltage of the transistor.
\end{abstract}

Keywords - MOSFET, modeling, technology, VLSI

\section{INTRODUCTION}

The integrated chips performing difficult functions have found broad application in the electronic equipment, in particular, in electronic control systems of modern cars. Practically all electronic systems of cars are focused on work at the source voltage of its onboard network which for cars has the value of $+12 \mathrm{~V}$. It means, that most components of chips of automobile electronics have to function normally at the same voltage. At the same time modern standards demand quite severe requirements to the used element base. In particular, according to these documents the voltage of breakdown of the MOS transistor must exceed its operating voltage at least by 3 times.

For the reasons stated above the problem of design of chips whose elements have submicronic design norms is relevant and contain the peripheral high-voltage transistors which have the breakdown voltage of about 36$40 \mathrm{~V}$ and operating voltage of $+12 \mathrm{~V}$ in addition to the normal MOS transistors which work at the standard supply voltage of $+5 \mathrm{~B}$ and define the logic of work of digital VLSIs. This task turned out to be connected with the solution of the complex of a number of subtasks among which we should note the right choice of construction of the high-voltage MOS transistor and the definition of an optimum concentration of the profile of its areas.

\section{STRUCTURAL DESIGN OF LDMOS-TRANSISTOR}

The major indicator defining the choice of construction of the high-voltage MOS transistor is the simplicity of its implementation when it is embedded in the existing process of forming of VLSI at the minimum design norm of $0.35-0.5$ microns.

The analysis of the known constructions of the highvoltage MOS transistors considered in [1-3] showed that the construction of the so-called LDMOS transistor is most suitable for the existing process flow. Its characteristic design feature is the existence of an extended weakly-doped area which adjoins the drain region, which is called DRIFT-region that allows to create an impurity depletion zone on which length there is a uniform voltage drop (figure 1) at the expense of small extent of alloying.

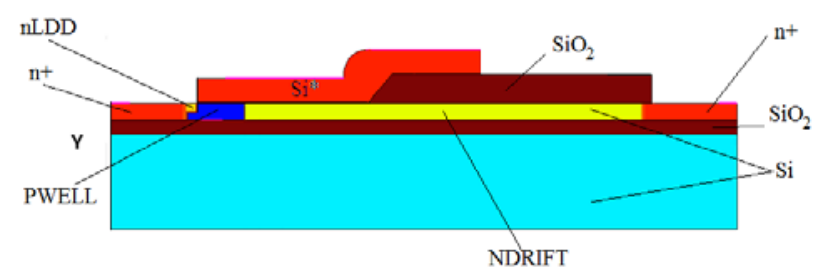

Fig. 1. The structure of the $n$-channel SOI LDMOStransistor with DRIFT-region

As a result it is possible to reduce significantly the electric field intensity in the whole DRIFT-region that, in turn, reduces the probability of emergence of avalanche effects leading to the breakdown of the MOS transistor.

Creation of the structure shown in figure 1 involved the solution of several practical tasks. First, it was necessary to define the concentration of doping impurity in DRIFT-region which would allow to reach the necessary puncture voltage and would provide normal functioning of the MOS transistor at the operating voltage of about +12 V. Secondly, for ensuring high breakdown voltage it was necessary to raise a stock on breakdown voltage of subbarrier dielectric by increasing its thickness in LDMOS transistors up to the value of about 150-200 $\AA$. Thirdly, it was necessary to provide forming of a thick layer of silicon dioxide over DRIFT-region. And, at last, fourthly, as the use of LDMOS transistors together with the transistors having normal operating voltage up to $+5 \mathrm{~B}$ is expected in the future, it was necessary to build in the technological process of LDMOS transistor forming into the existing technological process of VLSI production.

\section{TECHNIQUE OF CARRYING OUT CALCULATIONS}

In the represented work we speak about the solution of the problem of search and definition of the most preferable doping concentration in DRIFT-region at which the MOS transistor breakdown voltage would be 
maximum at the restrictions set for its geometrical sizes (the DRIFT-region length should not exceed 6 microns).

The following physical mechanisms of decrease in their mobility were taken into consideration: the dispersion on thermal fluctuations of crystal lattice atoms, the dispersion on impurity ions, the influence of a strong electric field, the dispersion on the roughnesses of the border subbarrier oxide-silicon. The calculations were carried out with the help of the TCAD system. The hydrodynamic model of electric charge carrier transfer was used.

As the calculation of MOS-transistors functional parameters at a high voltage on the drain assumes taking into account the effects of avalanche multiplication of the charge carries and it leads to a significant increase of time for the execution of calculations, the process of modeling was divided into two stages. At the first stage modeling parameters simulation of the MOS transistor construction simplified option was carried out. In this option it was supposed that all the areas had a strict rectangular form and all the concentration of the carriers within each region was strictly constant. The value of concentration in the field of alloying of pockets, LDD region and heavily doped regions of a source/drain was set by the constants which were received on the basis of the model of the existing technological process into which it was required to integrate the process of forming of LDMOS transistors. The thickness of subbarrier oxide was set to the constant equal to $150 \AA$ (the limited opportunities of the technology equipment requires the choice of this value). Oxide thickness over DRIFT-region was selected of such value that it was sufficient for masking DRIFT-region from alloying the region of sources/drains.

When calculating in the simplified option the following characteristics varied: impurity concentration in DRIFT-region; DRIFT-region extent (no more than 6 microns); MOS transistor channel length; amount of overlap between the thick oxide area and polysilicon layer; thickness of the SOI body; thickness of the buried oxide layer.

Using the Synopsys TCAD Sentaurus system the cycle of calculations for functional parameters modeling of the $n$-channel MOS transistor with the minimum design norm from 1.5 to 0.5 microns were carried out.

When modeling the possible misregistration of topological layers was taken into account, taking place because of the errors in the combination at the execution of lithographic operations. On the basis of the received results the norms on the geometrical extent of structure, in particular, the norm at the minimum length of the polysilicon area over thin oxide were defined: in the worst situation, with smaller lengths, DRIFT-region of alloying completely moved towards the thick oxide that led to the loss of LDMOS transistor management by means of gate MOSFET.

At the second stage, done in accordance with full value requirements, instrument and technological modeling construction of the transistor with the definition of the optimum modes of DRIFT-region ion implantation was carried out for the construction of LDMOS transistor which had the best value of puncture voltage and which met the requirement of operating voltage of $+12 \mathrm{~V}$. Before that, by means of the SIMS-analysis (SIMS - The secondary ionic mass spectroscopy method) the calibration of ion implantation process models was executed. It was shown that the Monte Carlo model reproduces the result of SIMS quite precisely.

Similar calculations were performed for p-type LDMOS transistors.

As a result, the recommendations on the structure and composition of the test chip necessary for characterization and forming process control of the LDMOS transistors operating at an increased supply voltage were developed on the basis of instrument-technological modeling.

\section{DISCUSSION OF THE RECEIVED RESULTS}

The calculations performed at the first stage used the TCAD instrument-technological modeling system showed that the dependence of the breakdown voltage of such an LDMOS transistor on the doping level of the DRIFTregion is not monotonic (see Figure 2). It is explained by the fact that for large degrees of doping, its presence is not effective from the point of view of increasing the breakdown voltage, since the depletion of most of its length does not occur. On the other hand, with a small degree of doping and limited sizes of the DRIFT-region, its complete depletion occurs even at low voltages, the drain-source, and it also does not contribute to an increase in the breakdown voltage

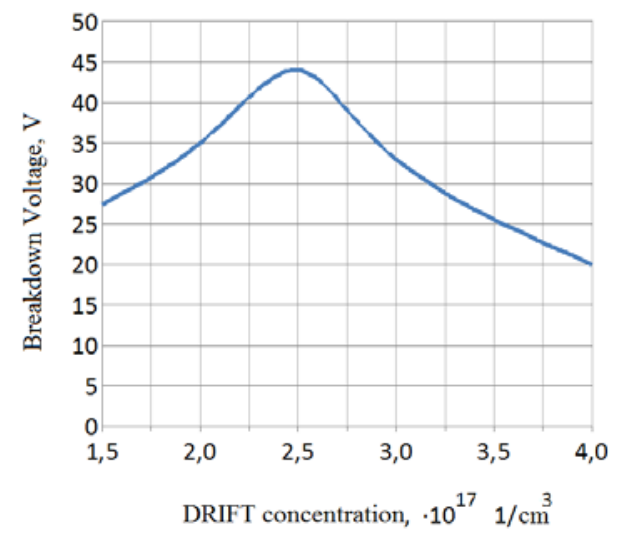

Fig. 2. Dependence of breakdown voltage on the impurity concentration in the DRIFT-region

The simulation results showed that the breakdown voltage practically does not depend on the amount of thick oxide area overlap with a layer of polysilicon.

It was found that MOSFET with a channel length of 1.5 microns and a DRIFT-region length of 6 microns, at the level of its doping about $2 \times 10^{17} 1 / \mathrm{cm}^{3}$ should have the greatest breakdown voltage about $40 \mathrm{~V}$. A weak 
dependence of the breakdown voltage on the length of the DRIFT-region was observed.

Before performing the second stage, by using the Monte Carlo method the models of well ion implantation process were preliminarily calibrated. Both the results of this calibration and the analytical model of the diffusion process of boron and phosphorus that exists in the TCAD system, are presented in Figures 3-6.

In the second phase, the full-fledged instrumenttechnological modeling with determination of the DRIFTregion ion implantation conditions was carried out for the MOS transistor with the highest breakdown voltage. As a result, the model of a MOSFET with an $n$-type channel was obtained, the calculated threshold voltage of which was $1.1 \mathrm{~V}$. Its set of calculated current-voltage characteristics is shown in Fig. 7, which shows, that there is no noticeable increase in the drain current at the voltage $0 \mathrm{~V}$ at the gate in the voltage range at the drain $0-40 \mathrm{~V}$. The rest of the characteristics were constructed at the gate voltages of $1.5-5 \mathrm{~V}$ with the step of $0.5 \mathrm{~V}$. The currentvoltage characteristics at the gate voltages of $0.5 \mathrm{~V}$ and 1 $\mathrm{V}$ are not shown, since these voltages are less than the calculated threshold voltage.

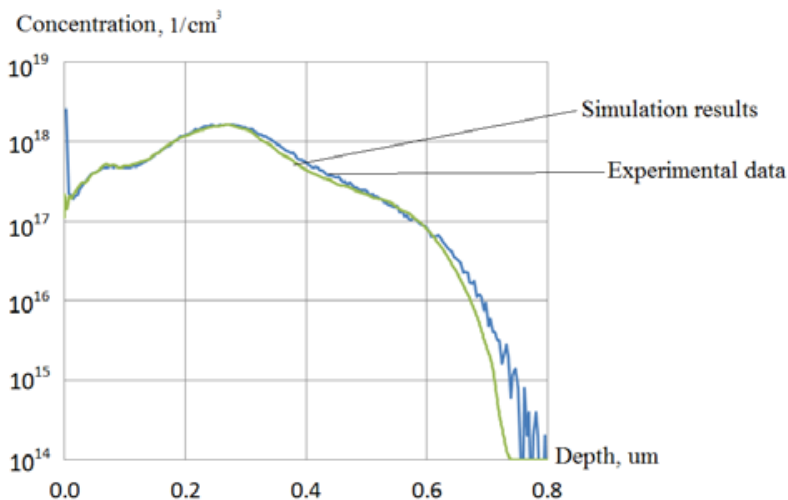

Fig. 3. The results of the calibration of boron ion implantation model

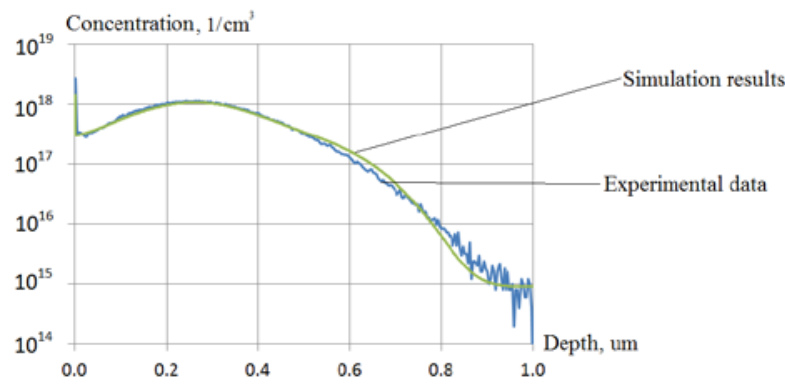

Fig. 4. The results of the calibration of the diffusion model of boron

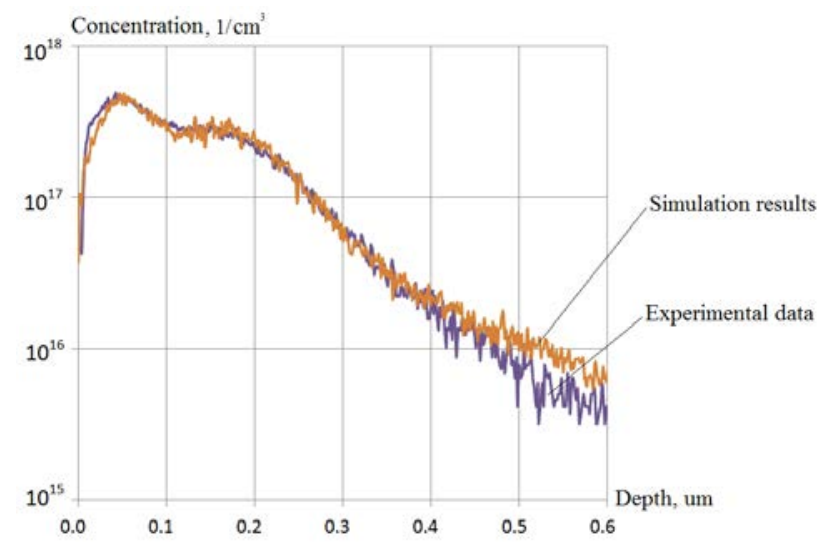

Fig. 5. The results of the calibration of the model of ion implantation of phosphorus

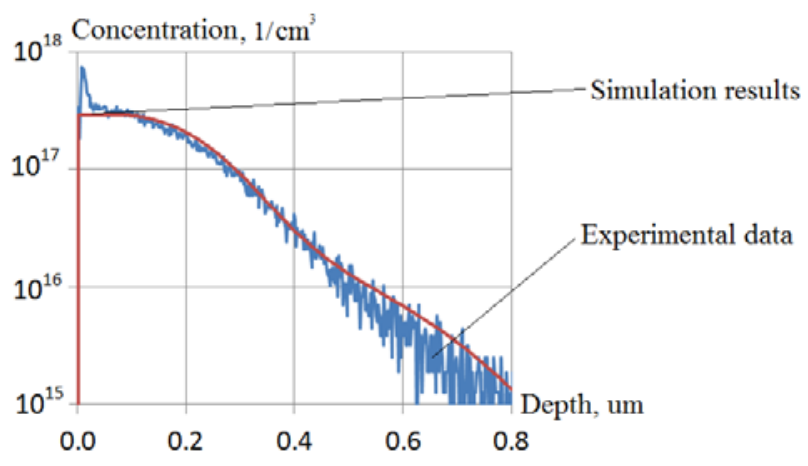

Fig. 6. The results of the calibration of the diffusion model of phosphorus

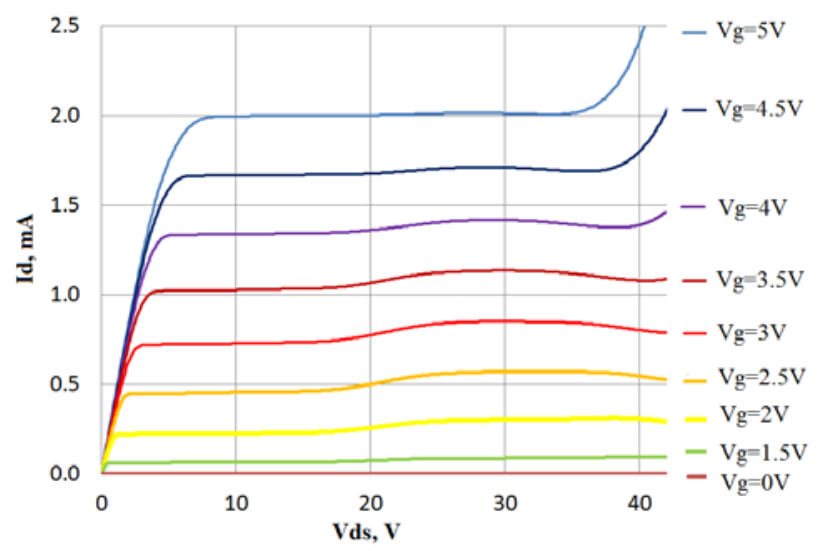

Fig. 7. Design set of current-voltage characteristics of LDMOS transistors with $n$-type channel

In order to analyze the reliability of the gate dielectric, the greatest potential in the near-surface layer under the gate oxide was determined. It turned out that it is approximately $7 \mathrm{~V}$. It means that in the worst case, $150 \AA$ thick sub-gate oxide should not be penetrated by the voltage between the working layers of silicon and polysilicon. 
A similar set of current-voltage characteristics of the p-channel MOSFET is shown in Figure 8.

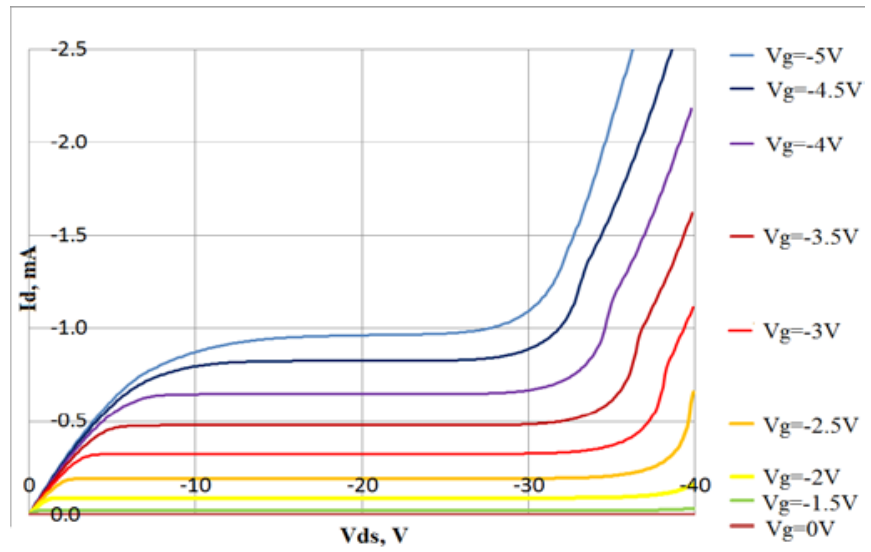

Fig. 8. Design set of current-voltage characteristics of LDMOS transistors with $p$-type channel

Figure 8 shows that at the specified doping concentrations of the DRIFT-region, the $p$-channel MOSFET can withstand slightly lower voltages (about 30 $\mathrm{V})$ in the open state.

Additionally, the distribution of the shock ionization rate in the LDMOS transistor structure was calculated and constructed (Figure 9).

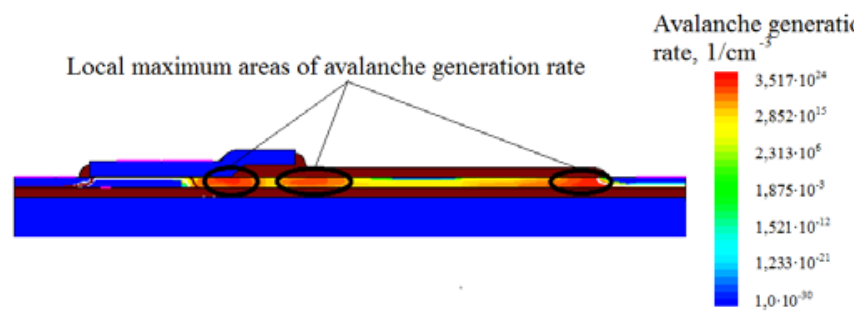

Fig. 9. The velocity distribution of impact ionization in the structure of LDMOS transistor with n-type channel

As figure 9 shows, the impact ionization rate has several local maxima, one of which is located near the transition of thin subatomic oxide into thick oxide over the DRIFT-region. It means that although the selected impurity concentration in the DRIFT-region allows to reach the maximum breakdown voltage it is not used effectively. By the way, it is proved by the weak dependence of the breakdown voltage on the length of the DRIFT-region

It is clear that to increase its efficiency it is necessary to reduce the concentration of impurities, which will reduce the breakdown voltage. It should be noted that at the first stage of modeling it has already been determined that the increase in the efficiency of the DRIFT-region without reducing the breakdown voltage is possible only by switching to the use of SOI-substrates which have greater thickness of the body silicon layer and buried oxide layer (thickness is about 0.5 microns or more).

\section{CONCLUSION}

The obtained simulation results were used to develop a technological route for manufacturing an experimental batch of plates, the parametric monitor of which contained a set of test structures, including conventional and high voltage $n$ - and $p$-channel MOSFETs. As a result of the experimental batch, the samples of $p$-channel MOSFETs were obtained, and they had a drain-source breakdown voltage of about $50 \mathrm{~V}$ and $n$-channel MOSFETs with drain-source breakdown voltage of about $40 \mathrm{~V}$.

Unfortunately, we did not manage to use effectively the DRIFT-region in the received structures. To increase its efficiency, it is necessary to use the substrates with large thickness of buried oxide and cut-off silicon (about $0.5 \mu \mathrm{m})$. In conclusion, we should note that the use of such substrates will require a radical revision of the entire technological route of VLSI formation.

\section{SUPPORT}

The work is executed at partial financial support of RFBR grant 16-07-00676 A.

\section{REFERENCES}

[1] Tkachyov A.U. The influence of design and technological factors on the electrical parameters of high-power LDMOS transistors. For the degree of candidate of technical Sciences, Voronezh, 2011, $159 \mathrm{p}$.

[2] J.M. Park, R. Klima, S. Selberherr. High-Voltage lateral trench gate SOI-LDMOSFETs // Microelectronics Journal. v. 35. 2004. pp. 299-304.

[3] JIA-FEI YAO et al : Low specific on-resistance SOI LDMOS device with $\mathrm{p}^{+-}$-p-top layer in the drift region / Jia-Fei Yao, Yu-Feng Guo, Guang-Ming Xu, TingTing Hua, Hong Lin, and Jian Xiao // Journal of semiconductor technology and science, Vol.14, No.5, 2014. pp. 673-681. 